\title{
El Ngillatun como sistema conceptual mapuche*
}

\author{
The Ngillatun ritual as a system of Mapuche symbolic concepts
}

María Catrileo

Universidad Austral de Chile, Facultad de Filosofía y Humanidades, Valdivia,

Chile. Correo electrónico: mcatrile@uach.cl

Este trabajo intenta describir el comportamiento etnolingüístico mapuche en una situación comunicativa que ocurre en la ceremonia ritual conocida con el nombre de Ngillatun. He utilizado la observación participante como procedimiento de recolección de datos, y el análisis componencial del concepto genérico de Ngillatun, incluyendo los rasgos lingüísticos y semánticos de términos discretos que simbolizan eventos tales como la convocatoria, los participantes, las visitas, la súplica, los bailes, los alimentos, los acuerdos y las plegarias de finalización. El resultado de este trabajo constituye una taxonomía que representa el sistema conceptual involucrado en este ritual mapuche. Asimismo, las conclusiones dan cuenta de una situación comunicativa en que un grupo de hablantes mapuche manifiesta su competencia nativa desde el punto de vista lingüístico y cultural.

Palabras clave: Ngillatun, términos discretos, taxonomía y competencia nativa.

This essay intends to describe the Mapuche ethnoliguistic behavior in the native ritual called Ngillatun. I have used participant observation as a data collection procedure, as well as the componential analysis of the genus term Ngillatun, including the linguistic and semantic features of cryptic terms involving events such as summons, participants, guests, plea, dances, food, common consent and closing prayer. This work displays a taxonomy representing the symbolic concepts that design the belief system involved in this Mapuche ritual. On the whole, it concludes that this is a communicative situation in which a group of Mapuche speakers makes use of the native communicative competence in a special linguistic and cultural context.

Key words: Ngillatun, cryptic terms, taxonomy and native competence.

El concepto genérico de Ngillatun posee rasgos lingüístico-semánticos propios y únicos. Sin embargo, este definendum comparte algunas características con otros miembros de su clase, lo cual nos permite descubrir una o más diferencias que indican

Este trabajo forma parte del proyecto FONDECYT Regular 2012 "La impronta andina en el sistema religioso cosmovisionario mapuche-williche”, Investigador Responsable Rodrigo Moulian T.; Coinvestigadora María Catrileo Ch. 
su definición propia. El grupo mapuche conserva varios rituales que mantienen ciertas semejanzas de forma y contenido en su realización. En la actualidad, la denominación de Ngillatun como signo lingüístico tiene variantes tales como Kamarikun, Llellipun y también Lepün en áreas de la Décima Región de Chile.

El concepto genérico de Ngillatun posee rasgos lingüístico-semánticos propios y únicos. Sin embargo, este definendum comparte algunas características con otros miembros de su clase, lo cual nos permite descubrir una o más diferencias que indican su definición propia. El grupo mapuche conserva varios rituales que mantienen ciertas semejanzas de forma y contenido en su realización. En la actualidad, la denominación de Ngillatun como signo lingüístico tiene variantes tales como Kamarikun, Llellipun y también Lepün en áreas de la Décima Región de Chile.

Este trabajo es el resultado de una selección deliberada de componentes del Ngillatun que reflejan parte del conocimiento cosmovisionario de los mapuche. La siguiente descripción se realiza en base al contexto en el cual aún se utilizan hoy, especialmente en áreas de la Novena Región de Chile. La metalengua empleada aquí es una forma de descripción para manejar y presentar la información etnolingüística registrada a través de nuestra observación participante en cuatro rituales celebrados en estos últimos años en el área de la IX Región de Chile: Huilío (1) (Barros Arana), Pitrahue (2) en las cercanías de Temuco, Rangintulewfü (3) en las cercanías de Nueva Imperial y Tromén (4) en los alrededores de Labranza.

He tomado como base teórica las tres dimensiones semióticas del signo lingüístico aplicadas por lingüistas y lexicógrafos en sus trabajos de investigación (Oller 1972; cf. Hartmann 1983: 111). En primer lugar, la sintaxis o la relación existente entre los signos de la secuencia del enunciado; en segundo lugar, el componente semántico o la relación que existe con la noción indicada en el enunciado; y en tercer lugar, la pragmática que tiene relación con los participantes del contexto de habla o espacio situacional. La Figura 1 ilustra estos tres componentes. El método de análisis semántico es contrastivo, pues la comparación de los componentes culturales propios del Ngillatun en cada espacio geográfico es necesaria para su definición como concepto genérico. De este modo se puede lograr un equilibrio entre su definición y la amplitud global de su significación dentro del grupo.

El componente sintáctico del signo Ngillatun se puede ejemplificar en el orden secuencial de los elementos lingüísticos en relación a otros signos en el sintagma o modelo oracional reconocido por los hablantes nativos de mapudungun. El componente semántico tiene relación con el sistema de selección de los elementos aceptados dentro de este paradigma. El componente pragmático se refiere, en este caso, a la relación existente entre los participantes del contexto de habla, los elementos seleccionados y la información extralingüística. Enfatizaré este tercer componente en el desarrollo de este trabajo, considerando la pragmática como una disciplina que describe las características contextuales de la comunicación, incluyendo el origen, los usos y los efectos de los signos dentro de los modos de comportamiento en que ellos ocurren (cf. Hartmann 1983).

La Figura 1 ilustra los tres aspectos del signo lingüístico diseñado por Oller en 1972 y aplicado por Hartmann en sus trabajos lexicográficos. El modelo presenta una dimensión sintáctica u orden secuencial que se extiende en el eje de izquierda a derecha, i.e. la sintaxis; la dimensión semántica que representa el sistema de selecciones, se encuentra en el eje que se extiende desde la parte superior hacia la 
inferior; $y$, por último, la dimensión pragmática o contexto situacional se ubica en el eje que se desplaza de derecha a izquierda.

Al aplicar este modelo al enunciado mapuche Trawulepay che Ngillatuwe mew ('La gente se ha reunido en el sitio ceremonial') en una situación contextualizada, podemos afirmar que los mapuche conocen su origen, sus funciones y sus efectos. Se trata de un ceremonial ancestral que se realiza según las normas del grupo con la finalidad de solicitar, agradecer y negociar los beneficios que entregan las deidades y las calamidades que el grupo puede enfrentar en caso de agraviar a estas divinidades. Cada componente de este enunciado tanto en el nivel de la oración como en el nivel de la frase y la palabra, constituyen el patrón de la estructura gramatical adecuada para indicar la relación que el hablante tiene con su audiencia y el contenido de su mensaje. En este caso, el acto de habla Trawulepay che Ngillatuwe mew (Figura 2) indica una reunión sagrada específica en el sitio ceremonial del ritual conocido como

Figura 1. Las tres dimensiones semióticas diseñadas por Oller (1972), citado por Hartmann (1983: 111).

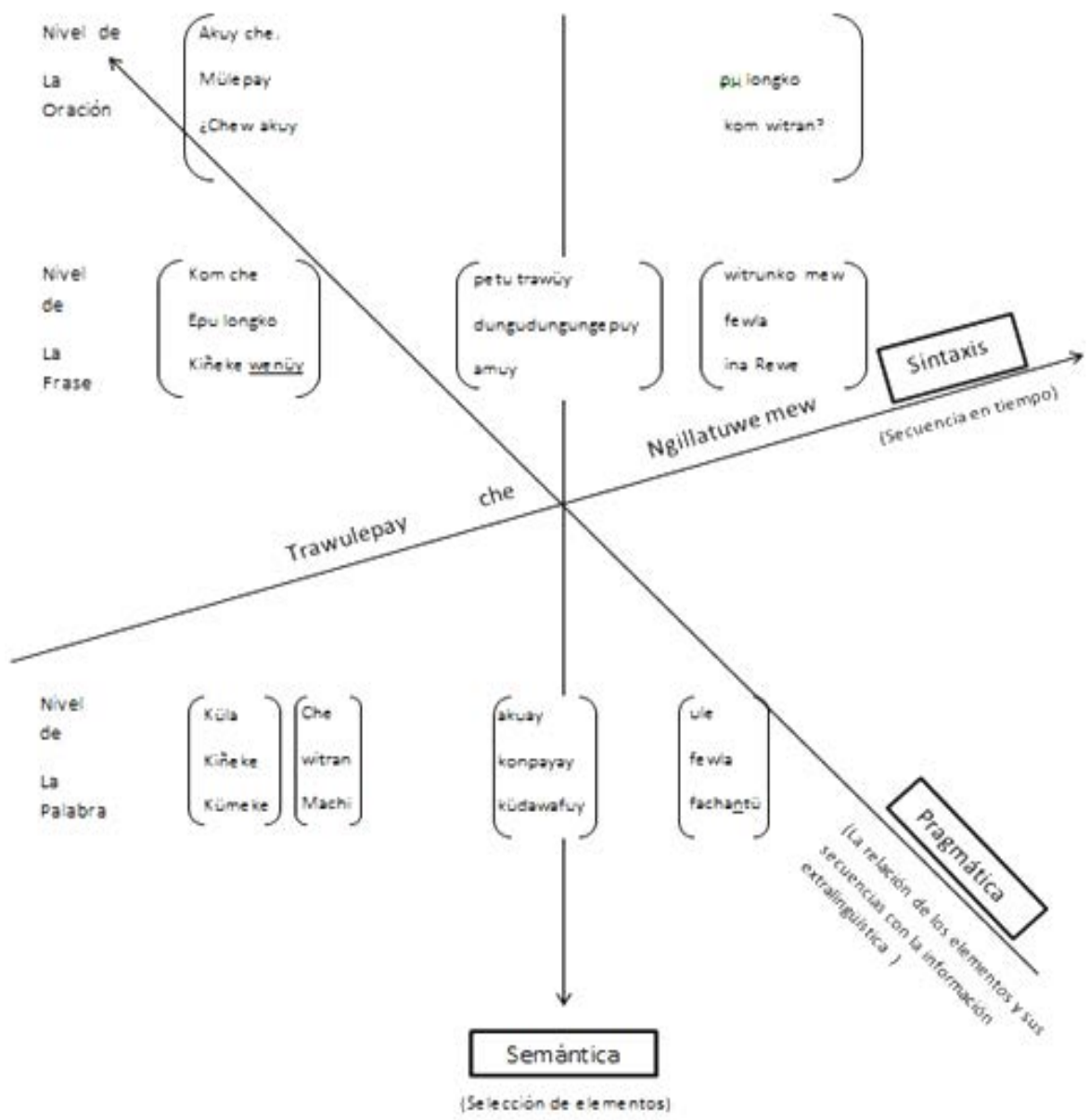


Ngillatun. Los elementos lingüísticos que componen esta oración están formados por frases y lexemas que constituyen la forma en que se hilvana el discurso textual para construir un enunciado coherente que permita la fluidez de la información. Los ítemes léxicos no funcionan aisladamente, pues la utilización de su forma y contenido depende de su ubicación en los diferentes tipos de discurso.

Trawulepay che Ngillatuwe mew puede tener una realización fonética diferente en la producción oral de cada persona que lo repite. Asimismo, su propósito comunicativo puede variar de acuerdo a su finalidad comunicativa, que puede incluir la simple descripción de una acción, un sentimiento de sorpresa o una respuesta esperada ante un estímulo anterior. Así, este acto de habla posee un potencial para informar o persuadir al interlocutor o al grupo para comportarse y actuar de una manera adecuada en la preparación y desarrollo del ceremonial en este caso.

El enfoque pragmático en torno al contexto nos ayuda a separar las características que señalan las posibles variaciones que ocurren en la celebración del Ngillatun en diversos espacios físicos habitados por el pueblo mapuche actualmente. La competencia comunicativa de los hablantes nativos incluye la habilidad para variar su modo de hablar de acuerdo a la situación en que se encuentran, como asimismo para reconocer y validar esta variación. La lengua como comportamiento gobernado por reglas en los contextos de interacción, permite a sus usuarios ejercer su competencia comunicativa dentro de su vida diaria. En concordancia con lo anterior y considerando el punto de vista lingüístico y denotativo, hemos intentado aplicar un enfoque basado en una matriz que muestra los parámetros de los componentes semánticos o de contenido que denotan el ceremonial de Ngillatun como un signo nativo vigente. Estos componentes pueden utilizarse como elementos de contraste en la comparación con otros rituales que celebra el grupo mapuche en el presente.

Desde la perspectiva histórica, este ceremonial ha sido celebrado y referido en forma continua a través de los tiempos. Así lo documentan las crónicas y traducciones tales como las memorias del Cacique Pascual Coña (1974 [1930]) y los escritos de Martín Alonqueo (1979, 1985); como asimismo, dentro de la conceptualización involucrada en este ritual, es preciso destacar otros trabajos más recientes de los etnógrafos, antropólogos, escritores y diversos especialistas tales como Armando Marileo (2000), María Catrileo (1995, 2010), M. Ester Grebe (2002), Tom Dillehay (2007), Hugo Carrasco (2010) e Iván Carrasco (1992). Casamiquela (1964: 3839), remontándose al mito del diluvio Trengtreng y Kaykay narrado por diferentes cronistas, sostiene que un grupo de tres mapuche compuesto por: Francisco Ladino de Maquehue, al sur de Temuco; Nicolás Quintrel de Conoco, cerca de Cholchol, y Pascual Alchao de Bajo Imperial, basaron el origen del Ngillatun en el mito de Trengtreng y Kaykay, después del cual los mapuche celebraron su primer Ngillatun. Cuenta la leyenda que el sacrificio de un niño huérfano y la sangre de gallos y gallinas aplacaron las aguas que estaban en retirada.

El enunciado Ngillatun está compuesto por el lexema ngilla que Luis de Valdivia (1606) define como: "gillan, cobrar o pedir la paga, o otra cosa". Sobre la base de sus observaciones en terreno y los datos bibliográficos situados entre el final del siglo XIX y el siglo XX, Casamiquela incluye como conceptos equivalentes del Ngillatun los siguientes términos: kamarikun, Llellipun, Ngillatun Kawiñ, Ayllarewe, Trawun y también Villatun que, en su tiempo, este autor considera como "una de las deformaciones que deben ser definitivamente proscriptas". Sostiene que en Argentina, 
más específicamente en la provincia de Neuquén, se emplea preferentemente Ngillatun, Llellipun y Kamarikun. En Chile este ritual tiene la denominación de Ngillatun y también Kamarikun en el área cordillerana pewenche (cf. Sánchez 20012002) entre la VIII y la IX Región y Lepün en partes de la X Región (cf. Moulian 2005, 2012). El ceremonial ha introducido elementos externos en su realización durante las últimas décadas, especialmente en los grupos más aculturados en donde la práctica de la lengua nativa o mapudungun tiende a disminuir (cf. Caniguan 2008). Antiguamente, según los cronistas y la información de algunos ancianos, la utilización del mapudungun se destacaba como medio de comunicación con sus diversos recursos estilísticos prosódicos y formales propios del contexto situacional en todo el entorno comunitario.

Actualmente, existen unos pocos ancianos monolingües de su lengua nativa y la mayoría es bilingüe de mapudungun y español con diversos grados de fluidez en ambas lenguas. Las funciones y los objetivos del ceremonial no han variado, pues se mantiene como un ritual destinado a rogar por el bienestar del grupo, agradecer los beneficios recibidos, negociar y demostrar los frutos del trabajo y el esfuerzo a través de la presentación de las ofrendas de alimentos y el sacrificio ritual que se ubica en el Llangillangi (ofrendario) y alrededor del Rewe (tótem mediador entre los habitantes de la tierra y el Wenu Mapu 'tierra de arriba'). El sitio ceremonial mantiene su espacio ancestral, excepto en aquellos pocos lugares en los cuales el interés y la veneración dedicada al ritual ha declinado por razones de aculturación, división de terrenos heredados e influencias de creencias externas en el conjunto de costumbres y modo de vida de los grupos que han sufrido los mayores cambios por influencia externa (cf. Moulian). En este respecto, nuestros datos y observaciones en los sitios de Rangintulewfü de Boroa (3) y Tromen (4) de Labranza confirman este planteamiento. No obstante, en la última década, los Ngenpin o longko de estos grupos han decidido instalar nuevamente este ritual en espacios adquiridos para este propósito. Siguiendo la iluminación onírica de los pewma del Machi o el Ngenpin, el ceremonial se planifica ajustándose estrictamente a las normas antiguas de la simbología ritual, el ordenamiento de los elementos seleccionados, la ubicación de las familias anfitrionas, los grupos invitados y también las personas no convocadas a esta celebración. Los encargados de la organización tienen la responsabilidad de revisar y controlar la mantención de la formalidad y el respeto hacia todos los componentes culturales involucrados en este ceremonial. Según la información de los ancianos, en la antigüedad sólo los mapuche concurrían a esta ceremonia. Sin embargo, al parecer, una vez instaladas las instituciones, junto con el contacto lingüístico y cultural a lo largo de la frontera del conflicto mapuche-no mapuche, también se aceptó, paulatinamente, la concurrencia de algunos outsiders 'ajenos al grupo' a los rituales sagrados como el Ngillatun y el Machitun (cf. Núñez de Pineda y Bascuñán 1973; Archivo Nacional 1673). Por otra parte y sólo ocasionalmente, se observan visitantes no-mapuche dentro del sitio ceremonial. En lugares de la IX Región, éstos deben permanecer fuera del ámbito más privado del ritual, tal como ocurre en Rawe y Pitrahue en las inmediaciones de Temuco y Huilío en el área de Barros Arana, aun cuando en este último sitio ya se observan más participantes externos.

La matriz de la Figura 2, basada en el modelo de análisis diseñado por Hartmann (1983), muestra seis parámetros de rasgos diferenciadores que abarcan la amplitud semántica del concepto de Ngillatun, ilustrando así algunos de los caracteres 
pragmáticos más importantes de este ritual en su contexto. Además, éstos nos orientan en la identificación de las variaciones que presenta el mismo ritual en diversas áreas geográficas en donde aún se realiza. El enunciado ngillatun se origina del lexema ngilla +_tu_ 'marcador de reciprocidad y reiteración colectiva' + el sustantivador _n_: ngillatun que indica una ceremonia para rogar, agradecer y negociar colectivamente y en forma reiterada entre el grupo y el conjunto de los ancestros y las divinidades creadoras. Esto demuestra la existencia y práctica de la dualidad sagrada y cíclica considerada como un valor primordial en el grupo. Dentro de las funciones del Ngillatun, es preciso destacar, en primer lugar, el Ngülamtuwun o reunión íntima y de recogimiento en la cual un Machi o un Ngenpin entrega un mensaje de consideración, reconciliación, obediencia y mantención del legado cultural pactado entre las deidades, los ancestros, la naturaleza y los habitantes del Мари. La existencia de la visión onírica en los cuatro rituales observados indica el punto inicial del ceremonial. El pewma cumple un papel importante en el sentido de permitir al Machi o chamán realizar sus capacidades sobrenaturales para emprender

Figura 2. Análisis componencial del Ngillatun 2000-2013

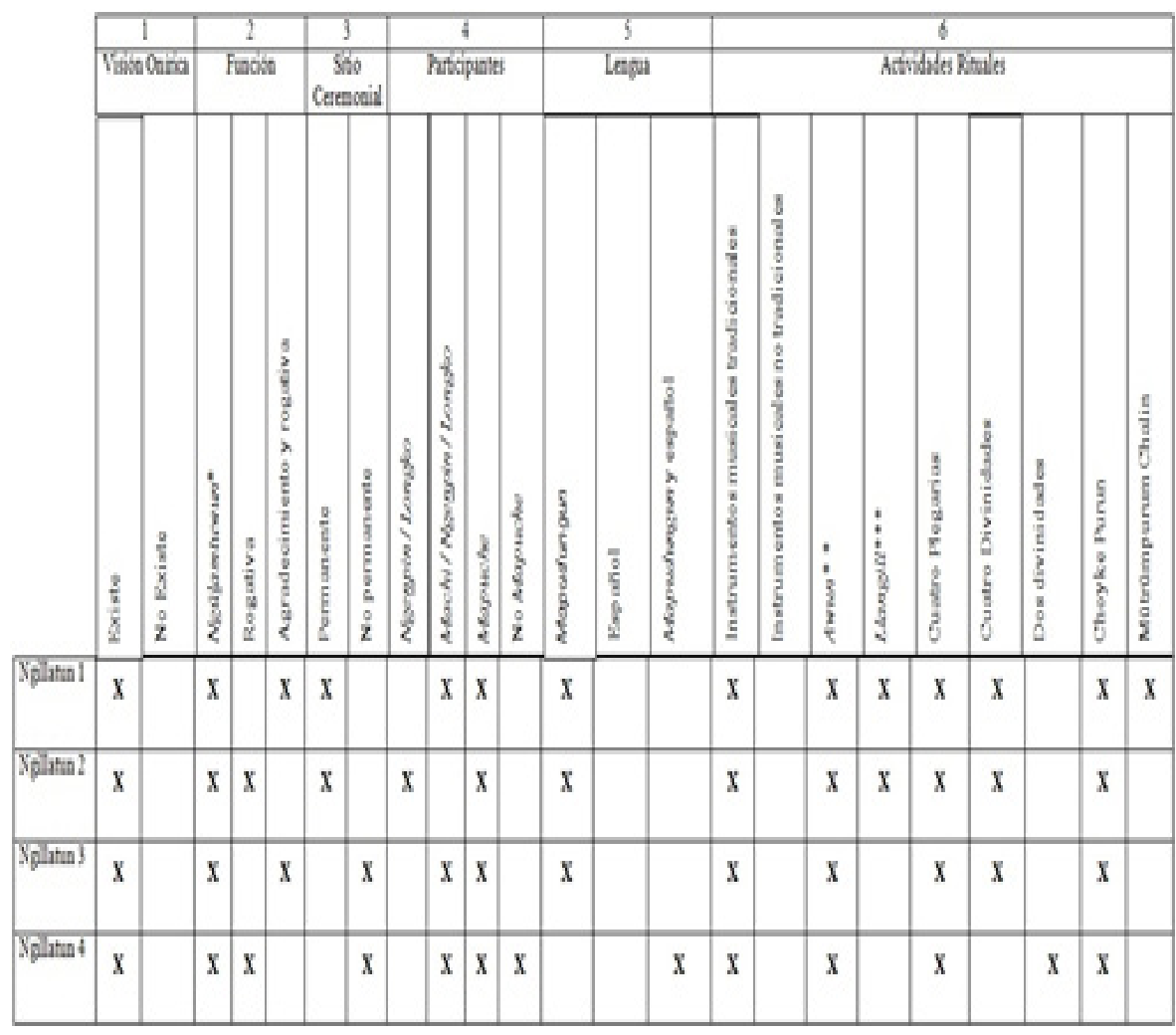

* Sesión sagrada de consejería y propiciación para el Ngillatun

${ }^{* *}$ Limpieza del sitio ceremonial realizada por corridas a caballo

${ }^{* * *}$ Sacrificio ritual de un animal o ave depositada en el Llangillangi (altar sagrado) 
su viaje al Wenu Mapu y mediar la comunicación entre Ngünechen y los habitantes del Mapu. La función del Ngillatun, considerando el Ngülamtuwun es, en primer lugar, agradecer y fortalecer los lazos existentes entre las deidades, los ancestros y los seres vivientes junto a la naturaleza en su conjunto; también suplicar y rogar por la mantención de los valores espirituales legados por los ancestros, como asimismo por los medios de subsistencia armónica y equilibrada. En cuanto al sitio ceremonial dentro de la organización del espacio en la cultura mapuche, el vocablo mapuche Ngillatuwe, formado por el lexema ngillatu 'pedir o solicitar con ruego colectivo y reiterado' + _we 'marcador de espacio o instrumento' indica 'el sitio en el cual se realiza la ceremonia de rogativa'. Este término designa un espacio físico sagrado que pertenece a la colectividad que lo instaló con una finalidad específica. Aquí se fijan los límites de creencias culturales que reflejan la organización social de la comunidad junto a la comunicación ritual con las deidades y los ancestros. Los participantes incluyen caracteres observables en general, tales como la ubicación, el vestuario, los colores y la presentación personal; asimismo, el tipo y la relación de roles que cada uno cumple dentro del grupo, las instituciones sociales representadas, las familias, las autoridades, sus jerarquías, sus derechos y responsabilidades dentro del ritual. La lengua dentro del ciclo de vida es muy importante, por lo que los Ngenpin, los Longko mayores y Machi lamentan en sus plegarias la pérdida paulatina del mapudungun en niños y jóvenes, pues este medio de comunicación nativo ya no posee un rol específico en el control social. El código verbal “mapudungun” y el código no-verbal utilizado en los componentes del mensaje junto a los eventos comunicativos en el ritual son relevantes para la rememoración y la mantención de los valores y la identidad del grupo. En esta situación ritualizada, los eventos incluyen actividades cuyo contexto está seriamente codificado en lo referente al rol de los líderes, los encargados de la música y los bailes, los instrumentos musicales permitidos, el encargado del Awun como ritual de limpieza del sitio ceremonial y la formalización de las cuatro plegarias en honor a las divinidades. El Tripal Ngillatun o evento de finalización, enfatiza la despedida y la exhortación final para la mantención del ritual, el cumplimiento de los nuevos compromisos adquiridos y el reforzamiento de los valores y los elementos culturales tradicionales.

Los parámetros de comparación incluidos aquí no muestran grandes contrastes entre los ceremoniales observados. Es importante señalar que el Perimontun Pewma 'visión onírica', el Awun, el Llangill, el choyke purun, los instrumentos musicales nativos y las cuatro plegarias se repiten en las áreas con menor grado de aculturación dentro de la sociedad mayor. No obstante, existe una ligera variación en la función que cumple el Ngillatun en las distintas localidades, pues, a veces, solamente se ruega por el bienestar del grupo, o, en otras ocasiones, se agradece y se propicia la mantención de los beneficios entregados por las deidades. Asimismo, es preciso destacar que en Rangintulewfü (3) y en Tromen (4) el ritual se realiza en un sitio no tradicional adquirido por la comunidad para los efectos de esta celebración religiosa, previo a un Trawun o reunión llevada a cabo entre las familias del lugar para conversar sobre la importancia de continuar la celebración de este ritual que, antiguamente, se realizaba en un sitio tradicional cercano. En estas localidades las familias tradicionales han permitido la participación ritual de personas no mapuche ya asentadas en estos lugares por motivos de unión conyugal o aceptación dentro del grupo. Esto no se observa en el área de Pitrawe en donde este ritual mantiene 
estrictamente la usanza tradicional dentro del sitio ceremonial, especialmente en lo referente a los participantes, la utilización del mapudungun, el sacrificio ritual y la mantención de los bailes e instrumentos musicales autóctonos, tales como el kultrung, la trutruka, la pifüllka y el kullkull. Por otra parte, en el ritual celebrado en Tromen (4), la referencia a dos divinidades, principalmente, mantiene la dualidad sagrada que, en este caso, representa a las cuatro deidades que conforman la familia Ngünechen. En lo referente al ritual del sacrificio o LLangill, éste se mantiene en los grupos más tradicionales, es decir, Huilío y Pitrawe. En Rangintulewfü y en Tromen no se ha observado.

El término Ngillatun puede constituir, teóricamente, la denominación genérica o común denominador para todas sus variaciones. En concordancia con lo anterior, no existe un modelo uniforme de este ritual en su realización de superficie. No obstante, sus cimientos subyacentes constituyen el conocimiento y el discernimiento nativo ancestral que se ha mantenido en un espacio-tiempo de gran extensión. Su contenido semántico se refiere a un concepto mental que, en español, se puede definir como un ritual mapuche consistente en el agradecimiento, la súplica y la negociación ante Ngünechen por los beneficios recibidos, además de la exhortación que se hace a los participantes para la mantención del ritual según el ordenamiento legado por los antepasados. En este sentido, las relaciones semánticas entre las diversas variaciones de Ngillatun celebradas en las diferentes localizaciones geográficas, están enlazadas por una derivación histórica común que varía en sus modalidades concretas de realización.

Durante los ceremoniales celebrados en lengua mapuche en la Novena Región, se repiten enunciados que cumplen la función de propiciar y mantener la identidad del grupo dentro de la sociedad mayor. Cada uno de sus miembros posee un repertorio de identidades sociales asociadas a formas de expresión verbal y no verbal aceptadas para la ocasión comunicativa en un contexto determinado que define y limita la interacción dentro del grupo. Así, en lo que Troike (1982: 181-2) denomina "sentido cultural”, el grupo mantiene las formas de comportamiento, las convenciones y los modos de las relaciones interpersonales adecuadas que sus miembros reconocen y practican en el ritual. El Ngillatun incluye eventos discretos que, desde el punto de vista etnolingüístico, constituyen sellos de ordenamiento, preparación, compromiso y responsabilidad formal dentro del desarrollo del ceremonial. En la actualidad, sus componentes se pueden describir de la siguiente manera a la luz de nuestra observación participante y las conversaciones sostenidas con algunos miembros pertenecientes a cada grupo considerado en este trabajo.

1. El Perimontun Pewma es un acto de representación de los anhelos y una señal o visión onírica de un Machi, Ngenpin o Longko interpretado como la predicción de un suceso, o la aparición de un espíritu benéfico que propicia la celebración del Ngillatun en el grupo. Un Ngenpin, Machi o Longko de la comunidad entrega esta vivencia a manera de información a los jefes y a las familias quienes obedecen la ordenanza del líder con la debida formalidad y respeto. De este modo, comienza el Ngülamtuwun o sesión sagrada de información, propiciación y exhortación mutua sobre la conveniencia de realizar el ritual.

2. El Elantün se refiere a la reunión especial para fijar la fecha y la duración de la ceremonia. Antiguamente, el Ngillatun duraba cuatro días en honor a las cuatro deidades representadas en Ngünechen. Actualmente, la mayoría de las comunidades 
dedica dos días a esta celebración ritual por razones socio-económicas, principalmente. Por lo tanto, se realiza a cada cuatro o dos años, según los acuerdos y necesidades del grupo. El Ngenpin (‘dueño del saber o jefe del grupo’) posee los conocimientos y la autoridad para realizar la convocatoria e invita al vecindario que incluye a todos los jefes de hogar, quienes tendrán la responsabilidad de acoger a las familias y amistades que acudirán al ritual desde otras localidades.

3. El Pepikawün se refiere a los preparativos previos a la ceremonia oficial. Los Ngenpin o Longko y los jefes de familia acuden al Ngillatuwe ('sitio ceremonial') con el fin de decorar o plantar el Rewe y el Llangillangi 'espacio del ofrendario, ruego y alabanza a Ngünechen'. Generalmente, en este lugar existen plantas permanentes como el aliwen ('tipo de manzano') y el külon o maki (aristotelia chilensis). También se adorna con ramas y hojas de aroma agradable como el triwe ('laurel') y los rüngi ('colihues'). Este espacio sagrado debe ubicarse en relación hacia la salida del sol o Puel Mapu, según la temporada correspondiente. Los días elegidos para el ritual deben coincidir con Llegün Küyen o luna de los brotes para propiciar el bienestar y el buen desarrollo de los seres humanos, las plantas y los animales del Mapu. Luego se llevan a cabo diversas actividades culinarias en los hogares con el fin de agasajar a los participantes. El muday es la bebida sagrada que todos los congregados deben ofrecer a las deidades y ancestros que también comparten el sitio ceremonial. Se entregan las instrucciones para la presentación personal, los atuendos y los símbolos rituales que se incluirán en esta ocasión. Según los propósitos de la rogativa, se recuerda a los participantes que deben utilizar determinados colores benéficos como el blanco y el azul de las banderas para agradar a las deidades de Wenu Mapu ('tierra de arriba') y así propiciar el buen tiempo y el bienestar del grupo. Las alhajas plateadas permitidas, las cintas y las vestimentas de todos los participantes deben seguir las normas de correcta presentación.

4. Los Kamañ Kona son las comisiones de trabajo ritual y la mantención del orden en el sitio ceremonial. Tienen distintas responsabilidades, tales como el traslado de los Machi y a Kallfümalen ('dama azul del canto sagrado'); el jefe de los Choyke ('bailarines con sus atuendos y adornos'); el Külliñ Kona ('encargado de la presentación y ubicación de los animales: ovino, ovejuno y aves') en el espacio ritual; los Sarkentu Kona ('hombres a pie con su Rüngi picana') o Kapitan ('hombres montados') que llevan la bandera de cada grupo junto a los adornos que representan a cada sector; los Awün Kona son los encargados de la demarcación del espacio de la troya o trilla a caballo; los Malwe Kona, encargados de los malwue o metawe ‘cántaro' de muday que se ubican en hilera frente al Rewe de norte a sur; las LLiwe malen ('encargadas de las pinturas de azul, blanco o negro') según el propósito de la rogativa, con el fin de reforzar la unidad del grupo; y por último, los kollong ('cuidadores del orden exterior') durante el desarrollo de las súplicas y los bailes alrededor del Rewe. El Adelkawe dungulfe kona ('encargado de preparar a los músicos y los instrumentos musicales'), tales como el tayüllfe ('acompañante del Machi en el kultrung'), el kull kull que se utilizará para los llamados al ritual, las trutruka, los dollkiñ y las pifüllka.

5. El Kimelchefe werken es el enviado especial para informar a los grupos externos convocados para este ceremonial. El Ngenpin ('poseedor del saber') envía a sus Werken o Kona ('mandatario') para entregar verbalmente la invitación en todos los sectores correspondientes. Este mensajero comenta los sueños y las visiones de los Machi, el Ngenpin y Longko, lo cual debe ser atendido adecuadamente para evitar calamidades futuras. Los convocados asumen su responsabilidad de participación afirmativamente y el Werken regresa para dar cuenta a las autoridades anfitrionas sobre la buena acogida de la convocatoria. El Ngenpin o Longko de las comunidades invitadas difunde el mensaje entre las familias y así comienza la preparación de los convidados para asistir al Ngillatun en el día señalado. 
6. El Amulnewenün es la propiciación del buen desarrollo de la ceremonia con el impulso de las fuerzas benéficas. En el día antes de su inicio y temprano en la mañana, el Ngenpin, el Longko y los encargados de la organización y la infraestructura del sitio ceremonial revisan la marcación del espacio y la ubicación exacta de los chellkom ('ramada') de cada Ngenruka ('jefe de hogar'), familia anfitriona o kamaska en el Lepün (cf. Moulian 2005) con la finalidad de asegurar la iniciación solemne del Ngillatun. El Longko principal junto al Ngenpin hacen izar las banderas blancas y azules que simbolizan el buen tiempo y la tierra azul de arriba. Las banderas negras se utilizan para favorecer la llegada de las lluvias. Luego el primer tañir de los instrumentos musicales simboliza la preparación, el recogimiento y la invitación para reunirse alrededor del Rewe y el Llangillangi. El Longko entrega las instrucciones correspondientes a los encargados de la organización general de la ceremonia, exhortándoles para actuar con responsabilidad en la mantención del orden y el debido respeto en el sitio ceremonial. El Longko recuerda a los participantes que este compromiso sagrado con Ngünechen se realiza para agradar también a los antepasados que cumplieron esta promesa con lealtad dentro de los plazos fijados. Por lo tanto, es un deber del grupo presente mantener y renovar este pacto con obediencia, sacrificio, unidad y humildad para agradar a Ngünechen. Luego los organizadores regresan a sus hogares para continuar con los preparativos de la ceremonia central que comenzará al día siguiente. Solamente los guardianes y los encargados de la música permanecerán en el sitio ceremonial para protegerlo y defenderlo de los espíritus negativos.

7. El Kon Ngillatun es el inicio de la ceremonia central en su parte vespertina. El Ngenpin, el Longko y su comitiva, los Machi y sus ayudantes se ubican al sur del Llangillangi. El resto de los participantes forman hileras para iniciar el baile ordenadamente conforme a las instrucciones, de norte a sur y de sur a norte.

8. El Awun es el rito de limpieza del sitio ceremonial y los vítores en homenaje a Ngünechen. Los representantes de cada familia acuden para integrar la caballería que galopará alrededor del Ngillatuwe hasta completar cuatro vueltas en honor a las cuatro divinidades del Wenu Mapu. A continuación, un grupo de varones realiza el Kefafan o ritual a voces en el altar sagrado para propiciar el llamado y la comunicación íntima con las deidades.

9. El Mütrümpurun Chalin es el rito de recibimiento de las visitas que en algunos ceremoniales como en Huilío se realiza a través del baile denominado mütrüm purun con gran júbilo y pasos que indican bienvenida, alegría y fraternidad. Los anfitriones y los invitados se mezclan entre los efusivos pasos de baile y juntos llegan hasta el centro del sitio ceremonial para tomar ubicación en el espacio correspondiente y así reiniciar el ritual junto al Rewe y el LLangillangi. Hombres y mujeres se ubican alternadamente frente a los Malwe o Marewpull Metawe ('cántaros rituales llenos de muday'). Al lado opuesto se ubican las visitas para participar en el Llitun purun ('baile solemne de cuatro tiempos'), acompañados con música y cantos de Machi. Toda la concurrencia, incluyendo a los kolle ('asistentes sin invitación'), deben participar en el baile.

10. El Purun incluye todos los tipos de baile realizados en la ceremonia. El llitun purun es el baile de inicio en donde, primeramente participa un grupo de choyke ('hombre avestruz que imita el baile de esta ave') se acerca al Rewe dando cuatro vueltas para saludar a las deidades y a las autoridades. El Tayülfe ('encargado del kultrung') tañe el instrumento según la orden entregada por el Machi. Los concurrentes bailan en hileras de hombres y mujeres en forma transversal y alternada, de norte a sur y de sur a norte guiados por los Purun Kamañ ('encargados del baile’).

11. El Llellipun es la invocación en que el Longko principal invita a los jefes de hogar a acercarse al Rewe y alzar sus malwe para escuchar al Machi y sus cuatro plegarias de agradecimiento, súplica y presentación de los productos del Mapu, dirigiendo los 
asperjes hacia los cuatro espacios de la tierra y hacia el Rewe conforme a las instrucciones entregadas por el Ngenpin. Luego se reanuda el baile de cuatro tiempos y, al finalizar, los participantes se detienen para compartir el muday. Los Machi realizan nuevas invocaciones y rogativas, haciendo un llamado de compromiso para ahuyentar influencias negativas y obedecer los mandatos de las deidades y los antepasados, manteniendo firmemente las costumbres tradicionales, los rituales, la lengua y el comportamiento adecuado en el grupo. Después continúa el baile hasta que el Ngenpin ordena su finalización.

12. El Ilelkawün es el agasajo que se ofrece a todos los participantes del ritual. Los Ngentrawün ('anfitriones') inician el Chalin ('saludo ritual') a los Mangel ('convidados que han tomado ubicación en los chellkom o ramadas'). Se retiran los mañumtun ('ofrendas desde el Rewe') y se sirven los alimentos. El alimento que no se consume se transforma en rokin ('merienda para el viaje') al regresar al hogar. Todos deben compartir el ágape y recibir un llawke ('porción de carne'), incluyendo a los kolletu ('personas no invitadas'). En la antigüedad, junto a la celebración del Ngillatun, existía un pequeño ceremonial llamado Konchotun, en el cual un varón pactaba amistad con otro varón, comprometiéndose a guardar esta relación de cercanía mediante un regalo que consistía en la entrega de un cordero como señal de amistad permanente. Actualmente, aún se observa un comportamiento similar en algunos grupos. Las nuevas amistades tienen el privilegio de ocupar los espacios y asientos adornados con mantas nuevas y colores adecuados para la ocasión.

13. El Tripal purun es el baile de finalización. El Ngenpin o Longko anuncia el baile final de despedida y un Machi se encarga de los tañidos del Kultrung. Los anfitriones y las visitas deben acercarse al Rewe para reanudar el baile a su alrededor hasta completar cuatro lukuntun ('arrodillarse') y cuatro vueltas rituales.

14. El Tripal Ngillatun es la etapa final de la ceremonia. El Ngenpin o Longko anuncia el término del baile y realiza la oración final. El discurso de despedida describe la actividad realizada, incluyendo los augurios del (la) Machi según los sueños del trance y la exhortación final para la mantención del ritual en el tiempo futuro. Así concluye la responsabilidad y el compromiso del grupo anfitrión en esta ceremonia. En forma simbólica se hace entrega del Rewe y los alimentos que están a su alrededor como una manera de asegurar la confianza en la realización y continuación del ritual en el tiempo correspondiente, según la tradición legada por los antepasados. El representante de las visitas asiente a esta demanda, comprometiéndose solemnemente a programar esta celebración en la fecha indicada.

\section{CONCLUSIÓN}

El resultado de este trabajo constituye una taxonomía que representa el sistema conceptual contenido en el Ngillatun. Esta situación comunicativa incluye eventos discretos que involucran a los participantes en un contexto específico y con un propósito definido que ocurre en la configuración general de actividades en una situación continua en que las formas simbólicas de expresión verbal y no verbal son siempre las mismas. Las reglas de interacción se repiten en el ceremonial y los participantes responden adecuadamente a cada uno de estos eventos comunicativos que son específicos de la cultura.

Las formas y contenidos lingüísticos representados por enunciados fijos dentro de la unidad global del Ngillatun cumplen la función comunicativa que se aprende en el grupo. De este modo, su realización verbal estimula la cooperación y la solidaridad entre un grupo de personas que comparten el mismo conocimiento cultural. Se trata de un concepto metafórico o fórmula lingüística que debe ser interpretada en un nivel 
abstracto. Así, los eventos discretos de este ritual poseen un sentido que depende de las creencias y los valores compartidos por los mapuche y no pueden ser interpretados en forma ajena a su contexto socio-cultural. Su codificación se encuentra en los patrones comunicativos del mapudungun que, en este ritual, hemos definido en seis parámetros cuyas especificaciones y rasgos característicos involucran componentes espirituales, humanos y materiales en la visión onírica, la función del Ngillatun, el sitio ceremonial, los participantes, la lengua y las actividades rituales que, en su conjunto, han mantenido la identidad del grupo a través del tiempo.

\section{OBRAS CITADAS}

Alonqueo, Martín. 1979. Instituciones Religiosas del Pueblo Mapuche. Santiago de Chile: Pontificia Universidad Católica de Chile. 1985. Mapuche ayer-hoy. Padre Las Casas, Chile: Imprenta y Editorial "San Francisco".

Caniguan, Jaqueline. 2008. "Variantes del mapuzugun”. Perfil sociolingüístico de comunidades mapuche de la VIII, IX y X Región. Santiago de Chile: CONADI-UTEM. 108-117

Carrasco, Hugo. 2010. "Discursos y metadiscursos mapuches”. Estudios Filológicos 45: 9-22.

Carrasco, Iván. 1992. "Un metatexto etnoliterario de los mapuches de Chile". Lengua y Literatura Mapuche 5: 183-192.

Casamiquela, Rodolfo M. 1964. Estudio del ngillatun y la religión araucana. Argentina: Instituto de Humanidades, Universidad Nacional del Sur.

Catrileo, María. 1995. Diccionario Lingüístico Etnográfico de la Lengua Mapuche. Santiago de Chile: Editorial Andrés Bello.

2010. La lengua mapuche en el siglo XXI. Valdivia, Chile: Universidad Austral de Chile.

Coña, Pascual, 1974. Memorias de un cacique mapuche (Transcrita en mapudungun y traducida al español por el P. Ernesto Wilhelm de Moesbach). Santiago de Chile: ICIRA.

Dillehay, Tom. 2007. Monuments, empires and resistance. The Araucanian Polity and Ritual Narratives. Cambridge: Cambridge University Press.

Grebe, M. Ester. 2002. "Los Mapuches de Chile en el Contexto del Mundo Andino: Algunas Perspectivas Interculturales”. Lengua y Literatura Mapuche 23-34.

Hartmann, R.R.K. 1983. Lexicography: Principles and Practice. London-New York: Academic Press.

Marileo, Armando. 2000. "Mundo Mapuche”. Luca Citarella, comp. Medicinas y culturas en la Araucanía: Santiago de Chile: Editorial Sudamericana. 91-107.

Moulian, Rodrigo. 2005. Tiempo de Lepün. Valdivia, Chile: Ediciones Universidad Austral de Chile.

. 2012. Metamorfosis ritual. Desde el Ngillatun al Culto Pentecostal. Valdivia de Chile: Consejo Nacional de la Cultura y las Artes-Universidad Austral de Chile.

Núñez de Pineda y Bascuñán, Francisco. 1973 (Archivo Nacional 1673). Cautiverio feliz. Santiago de Chile: Editorial Universitaria.

Oller, J.W. 1972. "On the relation between syntax, semantics, and pragmatics". Linguistics 83: 43-55.

Sánchez, Gilberto. 2001-2002. “Oraciones Rituales en Pewenche chileno Ritos ngillatun y püntevün”. Revista Chilena de Antropología 16:132-165.

Saville-Troike, Muriel. 1982. The ethnography of communication. An Introduction. Oxford: Basil Blackwell.

Valdivia, P. Luis de. 1606. Arte y gramática general de la lengua que corre en todo el Reyno de Chile, con un vocabulario y confesionario. Lima: por Francisco del Canto. 